\title{
Clinical value of vestibular-evoked myogenic potential tests in patients with sudden sensorineural hearing loss
}

\author{
Yuan Wang ${ }^{1 *}$, Shun-Tong $\mathrm{Gu}^{2}$, Xiao-Lin Bao ${ }^{1}$ and Jia-Liang Guo ${ }^{1}$
}

\begin{abstract}
Background: This study aims to investigate the clinical value of two kinds of vestibular-evoked myogenic potentials in patients with sudden sensorineural hearing loss (SSNHL).

Methods: A total of 82 patients were divided into two groups: vertigo group and non-vertigo group. All patients underwent examinations for pure tone hearing thresholds, middle ear analysis, the videonystagmography, caloric tests, and vestibular-evoked myogenic potentials elicited from the sternocleidomastoid and extraocular muscle. In addition, 30 healthy subjects were selected as the control group.

Results: For the 30 healthy subjects, the average latency of $\mathrm{p} 13$ and n23 of the cervical vestibular evoked myogenic potentials (cVEMPs) were $13.13 \pm 2.89 \mathrm{~ms}$ and $23.51 \pm 3.25 \mathrm{~ms}$, respectively, and the bilateral amplitude asymmetry rate ranged within $0.05-0.31$. The average latency of $\mathrm{n} 10$ of the ocular vestibular evoked myogenic potentials (oVEMPs) was $10.13 \pm 0.48 \mathrm{~ms}$. The average amplitude of the n10-p15-wave was $5.58 \pm 0.65 \mu \mathrm{V}$. Among the 35 vertigo patients with SSNHL, 27 patients had normal CVEMP and OVEMP examination results, five patients had abnormal oVEMP examination results, and five patients had abnormal cVEMP examination results. The latency and amplifier of oVEMPs and CVEMPs were within the normal range in 47 SSNHL patients without vertigo. The chisquare value was 5.647 , the $P$-value was equal to 0.017 , and the difference was statistically significant at a confidence interval of $95 \%$.
\end{abstract}

Conclusions: OVEMPs and CVEMPs can be used evaluate the vestibular nerve function of SSNHL patients with vertigo.

Keywords: Sudden sensorineural hearing loss, Vertigo, Vestibular evoked myogenic

\section{Background}

Sudden sensorineural hearing loss (SSNHL), namely sudden deafness, is a common disease of the ear, nose and throat (ENT), which mostly happens unilaterally, and sensorineural hearing loss with unknown cause (i.e. idiopathic), manifesting as different degrees of severity up to complete deafness. The Otolaryngology Head and Neck Surgery Branch of the Chinese Medical Association revised the diagnostic criteria for sudden hearing loss in 2015 [1]: SSNHL onset may occur without apparent cause, at least in the adjacent two frequency hearing

\footnotetext{
*Correspondence: wangyuan_wy33@163.com

'Department of Otolaryngology-Head \& Neck Surgery, Tianjin TEDA Hospital, Tianjin, China

Full list of author information is available at the end of the article
}

loss of $\geq 20 \mathrm{dBHL}$, and reaches its peak in $72 \mathrm{~h}$. Vertigo and/or tinnitus may additionally appear [2]. Although vascular, viral, or multiple etiologies have been proposed as causes of SSNHL, up to $90 \%$ of SSNHL cases are considered idiopathic [3]. Vertigo may develop in approximately 30-60\% of patients with SSNHL [4]. SSNHL accompanied with vertigo is usually considered to be caused by vestibular involvement. The vestibular-evoked myogenic potential (VEMP) is formed by myogenic responses activated by acoustic, galvanic, or bone vibration stimulation, and this is recorded by surface electromyography [5]. Cervical VEMP (cVEMP) was first introduced by Colebatch et al. The aim of this method is to assess the myogenic activity of the sternocleidomastoid muscle (SCM) [6]. Up to its advent, it was impossible to evaluate the otolithic organs [7-10]. 
In recent years, studies have shown that the cVEMP response to balloon and vestibular nerve function, while ocular VEMPs (oVEMPs) can respond to the upper vestibular nerve. The combination of these two tests may be able to respond to the vestibular nerves function in patients with SSNHL. Hence, the aim of this study was to evaluate the clinical value of two kinds of vestibularevoked myogenic potentials in patients with SSNHL. The results are presented and summarized as follows.

\section{Methods}

\section{Subjects}

According to the diagnostic criteria for SSNHL, the Chinese Journal of Otorhinolaryngology Head and Neck Surgery in 2015, and the vertigo symptoms, a total of 82 SSNHL patients were divided into two groups. Patients with vertigo were assigned to the vertigo group, while patients without vertigo were assigned to the non-vertigo group. All patients in the vertigo group exhibited nystagmus and the pattern of nystagmus did not change during the disease course. Patients with relapsed vertigo were excluded. All patients were unilateral and had no diseases, such as benign paroxysmal positional vertigo, labyrinthitis, vestibular neuritis, drug toxicity vertigo, vertebrobasilar insufficiency, and intracranial space-occupying lesions. A total of 30 healthy subjects with normal hearing and without vertigo were selected and assigned as the control group.

All subjects underwent the necessary physical examination, neurological examination, necessary laboratory test, and temporal bone computed tomography (CT) scan. And the pure tone hearing threshold, middle ear analysis, video nystagmography, caloric test data, cVEMPs and oVEMPs were also obtained.

\section{Caloric testing}

Caloric testing was performed by irrigating the external auditory canal with $2 \mathrm{ml}$ ice water $\left(4^{\circ} \mathrm{C}\right)$ for $20 \mathrm{~s}$ followed by aspiration of water. Caloric nystagmus was recorded, in a darkened room, using an electronystagmograph. We defined an abnormal caloric response by either of the following criteria: (1) canal paresis (CP) percentage $>20 \%$; (2) maximum slow phase eye velocity $<10 / \mathrm{s}$ bilaterally.

\section{Stimuli and recording techniques of CVEMPs [11]}

A commercial electromyographic (EMG) system (MEB$9200 \mathrm{~K}$, Japan) was used for the cVEMP test. Patients were tested in a sitting position during the cVEMP procedure. The disc-shaped recording electrode was placed at the midpoint of the sternocleidomastoid muscle (SMC) belly, a reference electrode was placed at the SMC clavicle, and a ground electrode was placed at the mid-forehead skin surface. Patients were asked to turn their heads contralaterally to keep the SCM muscle contracted. The skin surface impedance was $<5 \mathrm{k} \Omega$. Using the air guide click sound stimulation, the intensity was $105 \mathrm{dBnHL}$, stimulation frequency was three times per second, bandpass filter range was $500-2000 \mathrm{~Hz}$, and superimposition was 200 times. Sound stimuli were delivered monaurally and recorded bilaterally.

The latencies and amplitudes of the first positive-negative peaks (p13-n23) of the cVEMP were evaluated as described by Fujimoto et al. [12]. Briefly, the asymmetry ratio (AR) for p13-n23 amplitude (cVEMP AR) was calculated as $100[(\mathrm{Au}-\mathrm{Aa}) /(\mathrm{Aa}+\mathrm{Au})]$, where $\mathrm{Au}$ is the p13-n23 amplitude on the unaffected side and Aa is the p13-n23 amplitude on the affected side. On the basis of the results from normal subjects, the upper limit of the percentage cVEMP AR was placed. When no reproducible p13-n23 was present in 2 runs, we regarded it as an "absent response". When a reproducible p13-n23 was present and cVEMP AR (\%) was greater than the normal upper limits, we regarded it as a "decreased response".

\section{Stimuli and recording techniques of oVEMPs [13]}

A commercial EMG system (MEB-9200 K, Japan) was used for the oVEMP test. The patients were instructed to lie in the supine position. The standard was placed at $70 \mathrm{~cm}$ from the top of the head, with 25 degrees backward positioning. A disc-shaped recording electrode was placed at the middle of the lower side of the pupil at $10 \mathrm{~mm}$. The reference electrode was placed at the skin at $20 \mathrm{~mm}$ lower than that of the recording electrode. The ground electrode was placed at the middle of the forehead. The skin surface impedance was $<5 \mathrm{k} \Omega$. The air guide $500-\mathrm{Hz}$ click sound stimulation was employed, the intensity was $105 \mathrm{dBnHL}$. Sound stimuli were monaurally delivered and bilaterally recorded. Stimulus frequency was three times per second, bandpass filter range was $20-500 \mathrm{~Hz}$, and the superimposition was 50 times. The patients were prohibited from moving their head and instructed to keep their eyes on the visual testobject during the test. A significant negative waveform, named n10, was recorded at approximately $10 \mathrm{~s}$ after stimulus onset. The latency and amplitude of n10 were calculated. We analyzed the first negative peak (n10) latency, the subsequent positive peak (p15) latency, and the amplitude between $\mathrm{n} 10$ and $\mathrm{p} 15$. For the evaluation of amplitude, the asymmetry ratio for n10-p15 amplitude (oVEMP AR) was calculated as $100[(\mathrm{Au}-\mathrm{Aa}) /(\mathrm{Aa}+\mathrm{Au})]$, where $\mathrm{Au}$ is the n10-p15 amplitude on the unaffected side and $A a$ is the n10-p15 amplitude on the affected side. Responses recorded from the eye contralateral to stimulation were used for calculating oVEMP AR. On the basis of the results from normal subjects, the upper limit of normal oVEMP AR was set. When no reproducible n10-p15 was present in 2 runs, we regarded it as an "absent response". When a reproducible $\mathrm{n} 10-\mathrm{p} 15$ was present and the oVEMP AR (\%) was greater than the normal upper limits, we regarded it as a "decreased response" [12]. 


\section{Statistical analysis}

For statistical analysis, SPSS 17.0 software was used. The statistical results were presented as mean \pm standard deviation (SD). The chi-square was used to analyze the data between the two groups using the SPSS 17.0 software. The chi-square value was 5.647, the $P$-value was equal to 0.017 , and the difference was statistically significant at a confidence interval of $95 \%$.

\section{Results}

Among the 35 patients with vertigo, 16 patients had SSNHL in the left ear, while 19 patients had SSNHL in the right ear. Furthermore, among these patients, 21 patients were male, while 14 patients were female, and the age of these patients ranged within 23-49 years old. Vertigo almost simultaneously occurred at onset, without other nerve involvement symptoms. And the vertigo continued from one hour to five days. In addition, 32 patients were accompanied by nausea, while 16 patients were accompanied by vomiting. Among the 47 patients without vertigo, 23 patients had SSNHL in the left ear, while 24 patients had SSNHL in the right ear. 26 patients were male and 21 patients were female. And the age of these patients ranged within 21-46 years old. Among the 30 healthy subjects, 16 subjects were male and 14 subjects were female. And the age of these healthy subjects ranged within 22-48 years old (Table 1).

cVEMPs and oVEMPs were both evoked in the 30 healthy subjects. And the caloric tests were all normal.

Table 1 The characteristics of each group

\begin{tabular}{|c|c|c|c|}
\hline Characteristics & $\begin{array}{l}\text { Vertigo group } \\
(n=35)\end{array}$ & $\begin{array}{l}\text { non-vertigo group } \\
(n=47)\end{array}$ & $\begin{array}{l}\text { healthy subjects } \\
(n=30)\end{array}$ \\
\hline Age & $23-49$ years & $21-46$ years & $22-48$ years \\
\hline \multicolumn{4}{|l|}{ Sex } \\
\hline Male & 21 & 26 & 16 \\
\hline Female & 14 & 21 & 14 \\
\hline \multicolumn{4}{|l|}{ Side } \\
\hline Right & 19 & 24 & - \\
\hline Left & 16 & 23 & - \\
\hline \multicolumn{4}{|c|}{ Accompanying symptoms } \\
\hline Nausea & 32 & - & - \\
\hline Vomiting & 16 & - & - \\
\hline \multicolumn{4}{|c|}{ Severity of deafness } \\
\hline Mild & 6 & 6 & - \\
\hline Moderate & 21 & 29 & - \\
\hline Severe & 5 & 8 & - \\
\hline $\begin{array}{l}\text { Extremely } \\
\text { severe }\end{array}$ & 3 & 4 & - \\
\hline \multicolumn{4}{|c|}{ Caloric responses } \\
\hline Normal & 33 & 47 & 30 \\
\hline Abnormal & 2 & 0 & 0 \\
\hline
\end{tabular}

The latency of p13 and n23 of the cVEMP was $13.13 \pm 2.89 \mathrm{~ms}$ and $23.51 \pm 3.25 \mathrm{~ms}$, respectively. The amplitude asymmetry ranged within $0.05-0.31$. The latency of the $\mathrm{n} 10$ waveform of the oVEMP was $10.13 \pm 0.48 \mathrm{~ms}$, while the mean amplitude of the n10-p15 waveform was $5.58 \pm 0.65 \mu \mathrm{V}$. The amplitude asymmetry between the two sides ranged within $0.05-0.35$.

Among the 35 SSNHL patients with vertigo, six patients had mild deafness, 21 patients had moderate deafness, five patients had severe deafness, and three patients had extremely severe deafness. 2 of the 35 SSNHL patients with vertigo showed abnormal caloric responses on the affected side. Furthermore, 30 patients had normal latency and amplitude of cVEMP and oVEMP, five patients had abnormal cVEMP (two patients had an extended p13 latency, one patient had an extended n23 latency, and one patient had extended p13 and n23 latencies), five patients had abnormal oVEMPs (three patients had a n10 extended latency, while two patients were not evoked). Figures 1 and 2 are representative CVEMP and oVEMP images in the vertigo group.

Among the SSNHL 47 patients without vertigo, six patients had mild deafness, 29 patients had moderate deafness, eight patients had severe deafness, and four patients had extremely severe deafness. All patients had normal caloric responses. The oVEMP and cVEMP waveforms and latency were in the normal range.

As shown in Table 2, abnormal results for cVEMP and oVEMP can be detected in $25.7 \%$ (5/35) of patients with vertigo, which was statistically significant, when compared to patients without vertigo $(p=0.017)$.

\section{Discussion}

SSNHL is a type of sensorineural deafness without apparent cause. The cause of SSNHL with vertigo is more complex, and clinical hearing loss is usually worse [14]. The probability of hearing recovery is also significantly lower, when compared to those without vertigo [15-17].

Studies have shown that SSNHL patients with vertigo are different from patients with Meniere's disease. This is mostly caused by the involvement of vestibular nerve afferent receptors, in which the symptoms of vertigo rapidly improves through the rapid establishment of the vestibular central compensatory mechanism. The function of the vestibular nerve is essential for the diagnosis and differential diagnosis of SSNHL with vertigo.

The cVEMP can be used to assess the saccular function and inferior vestibular pathway, while oVEMP can be used to evaluate the utricular function and superior vestibular pathway [18-20]. For patients with SSNHL, the cVEMP and oVEMP tests can be more effective responses to vestibular nerve function [21].

In the present study, 82 patients with SSNHL underwent cVEMP and oVEMP tests. Abnormal results for 


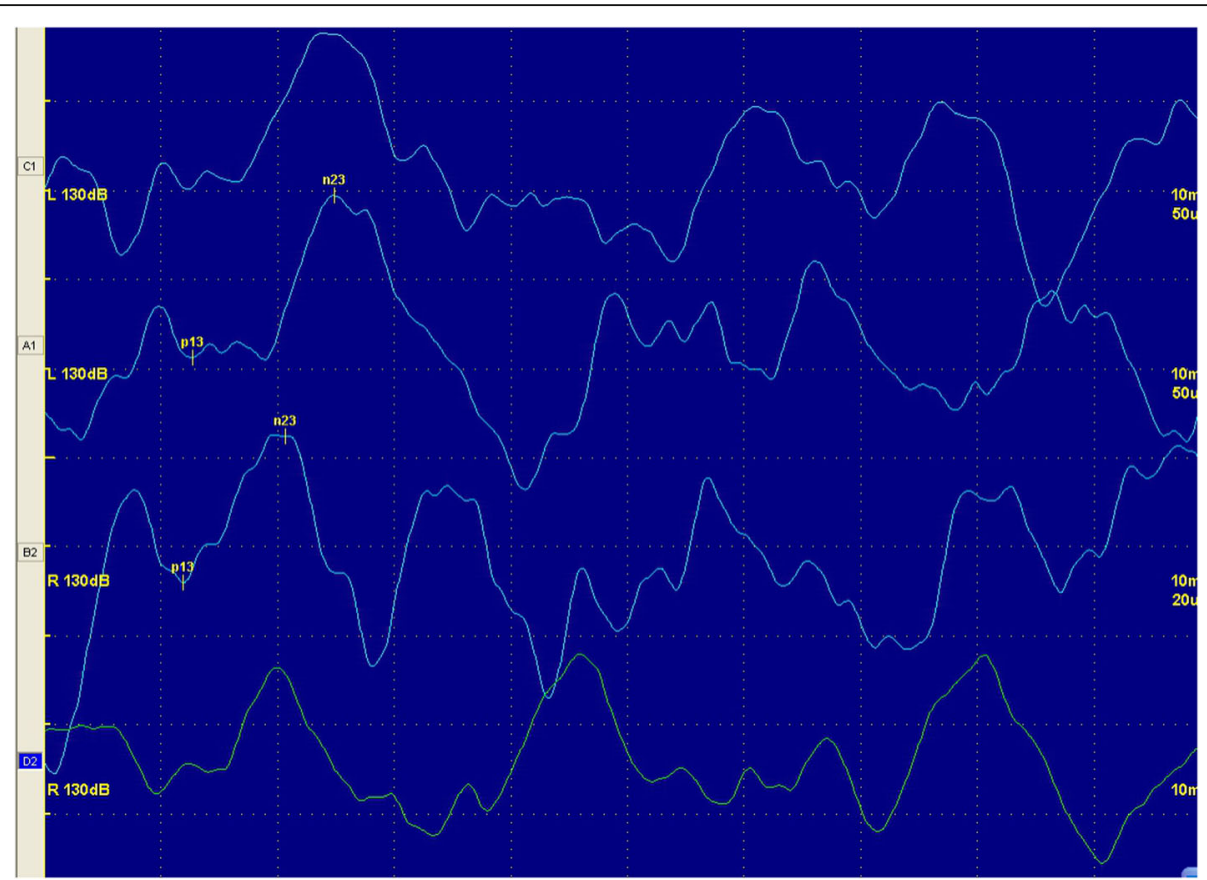

Fig. 1 CVEMP of a 30-year-old man in vertigo group. This patient showed extented latency of left ear, but showed normal cVEMP on right ear

cVEMP and oVEMP can be detected in $25.7 \%(9 / 35)$ of patients with vertigo, which was statistically significant, when compared to patients without vertigo. Hence, there is a certain clinical value to test the VEMP of SSNHL patients with vertigo. The abnormal rate of VEMP is high in patients with moderate to extremely severe deafness. Further observations are needed to determine the specificity of the diagnosis of vertigo. Moreover, more cases were needed to prove the significance of latency and amplitude before and after treatment.

From the abnormal rate of cVEMPs and oVEMPs, it can be concluded that the involvement of the upper vestibular nerve and lower vestibular nerve in SSNHL patients with vertigo were roughly similar, but the difference was not

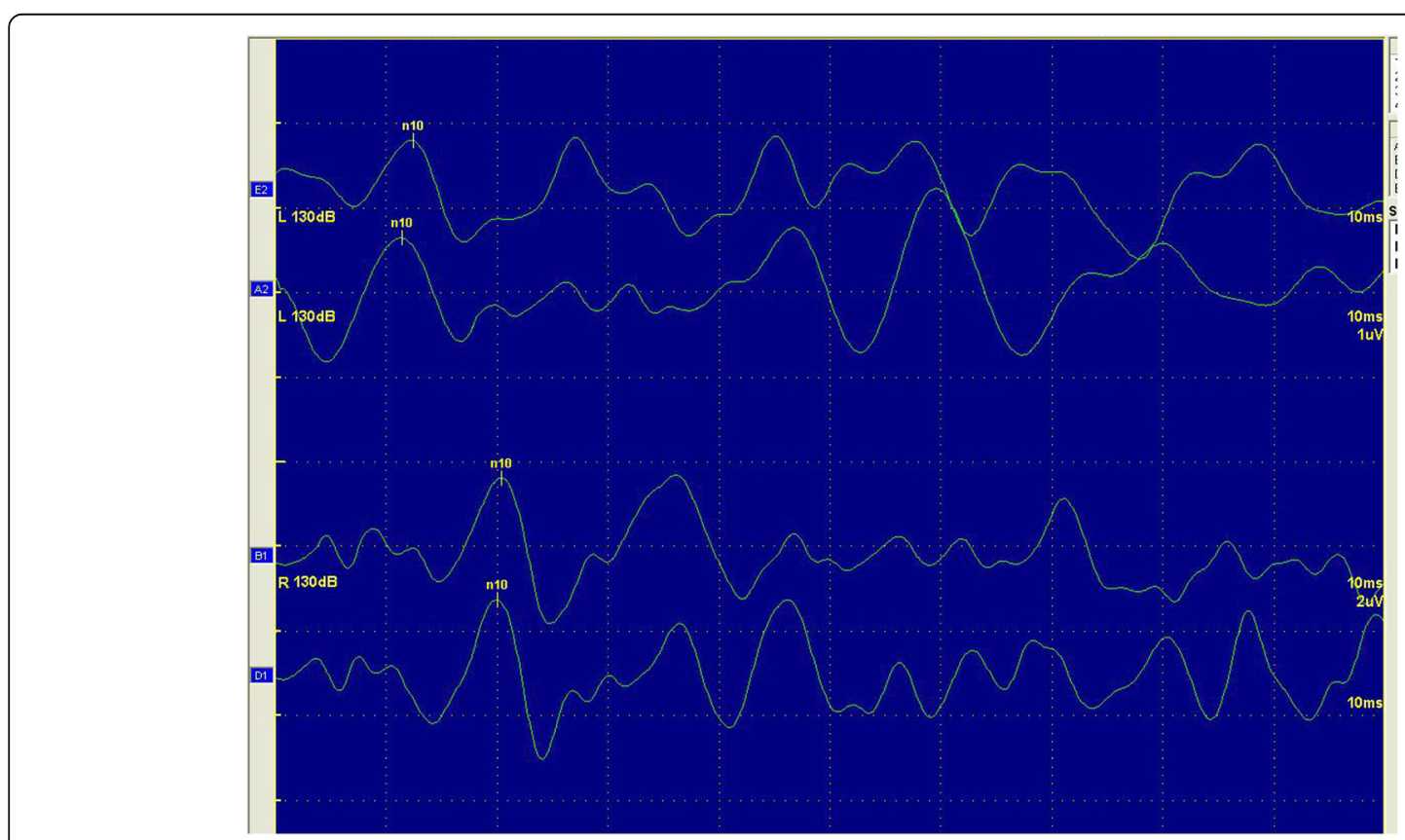

Fig. 2 OVEMP of a 42-year-old woman in vertigo group. This patient showed extented latency of right ear, but showed normal oVEMP on left ear 
Table 2 Analysis of association by the Chi-squared test according to the tests of CVEMPs and oVEMPs

\begin{tabular}{|c|c|c|c|c|c|c|}
\hline \multirow[t]{2}{*}{ Tests } & \multicolumn{2}{|c|}{ SSNHL with vertigo } & \multicolumn{2}{|c|}{ SSNHL without vertigo } & \multirow[t]{2}{*}{$x^{2}$} & \multirow[t]{2}{*}{$P$} \\
\hline & Abnormal & Normal & Abnormal & Normal & & \\
\hline cVEMPs & 5 & 27 & 0 & 42 & 5.647 & 0.017 \\
\hline OVEMPS & 5 & 27 & 0 & 42 & 5.647 & 0.017 \\
\hline
\end{tabular}

statistically significant. From the correlation analysis of cVEMPs and oVEMPs with pure tone and middle ear analysis, the chi-square test result was not statistically significant. Hence, it can be concluded that VEMP is not correlated to the degree of hearing loss and the functional status of the middle ear. Further studies are needed to determine whether VEMPs are correlated to cochlear electrograms, video electroencephalography, caloric tests and other vestibular function tests.

\section{Conclusion}

Sudden deafness is common in ENT, but the pathogenesis of patients with vertigo is more complex. Patients are at a substantially increased risk of vestibular organ lesions following a diagnosis of SSNHL, especially in the presence of vertigo [22]. For the initial onset of vertigo patients, it remains difficult to identify SSNHL accompanied with vertigo or Meniere's disease, which is caused by dizziness associated with hearing loss. Patients with SSNHL accompanied by vertigo have no specific findings with cVEMPs and oVEMPs tests. Hence, VEMP tests can provide evidence for the lesion location of the vestibular, and provide objective information for further clinical observation.

\section{Abbreviations \\ CP: canal paresis; CT: computed tomography; CVEMP: cervical VEMP; EMG: electromyographic; ENT: ear, nose and throat; OVEMPs: ocular VEMPs; SCM: sternocleidomastoid muscle; SD: standard deviation; SSNHL: sudden sensorineural hearing loss; VEMP: vestibular-evoked myogenic potential}

\section{Acknowledgements}

We would like to acknowledge the hard and dedicated work of all the staff that implemented the intervention and evaluation components of the study.

\section{Funding}

No external funding received to conduct this study.

\section{Availability of data and materials}

The datasets used and/or analysed during the current study are available from the corresponding author on reasonable request.

\section{Authors' contributions}

YW, STG acquired data. YW, STG and JLG drafted the manuscript. XLB and $J \mathrm{LG}$ contributed substantially to its revision. All authors takes responsibility for the paper as a whole. All authors read and approved the final manuscript.

\section{Ethics approval and consent to participate}

I confirm that I have read the Editorial Policy pages. This study was conducted with approval from the Ethics Committee of Tianjin TEDA Hospital. This study was conducted in accordance with the declaration of Helsinki. Written informed consent was obtained from all participants.
Consent for publication

Not applicable.

\section{Competing interests}

The authors declare that they have no competing interests.

\section{Author details}

'Department of Otolaryngology-Head \& Neck Surgery, Tianjin TEDA Hospital, Tianjin, China. ${ }^{2}$ Department of General Surgery, Tianjin Fifth Central Hospital, Tianjin, China.

Received: 21 October 2019 Accepted: 16 December 2019

Published online: 21 December 2019

\section{References}

1. Editorial Board of Chinese Journal of Otorhinolaryngology Head and Neck Surgery, Chinese Medical Association Otorhinolaryngology Head and Neck Surgery Science Branch. Guidelines for the diagnosis and treatment of sudden deafness. Zhong Hua Er Bi Yan Hou Tou Jing Wai Ke Za Zhi 2015,50: 443-447.

2. Deutsche Gesellschaft für Hals-Nasen-Ohren-Heilkunde, Kopfund HalsChirurgie. Leitlinie Hörsturz (Akuter idiopathischer sensorineuraler Hörverlust). 2014.

3. Stachler RJ, Chandrasekhar SS, Archer SM, Rosenfeld RM, Schwartz SR, Barrs DM, et al. Clinical practice guideline: sudden hearing loss. Otolaryngol Head Neck Surg. 2012;146:S1-35.

4. Rauch SD. Clinical practice. Idiopathic sudden sensorineural hearing loss. N Engl J Med. 2008;359:833-40.

5. Felipe L, Kingma $H$, Gonçalves DU. Potencial evocado miogênico vestibular. Arq Int Otorrinolaringol. 2012;16:103-7.

6. Colebatch JG, Halmagyi GM, Skuse NF. Myogenic potentials generated by a click-evoked vestibulocollic reflex. J Neurol Neurosurg Psychiatry. 1994:57:190-7.

7. Bansal S, Sahni S, Sinha S. Cervical and ocular vestibular evoked myogenic potentials in individuals with severe to profound hearing loss. J Hear Sci. 2013;3:56-63.

8. Fujimoto C, Suzuki S, Kinoshita M, Egami N, Sugasawa K, Iwasaki S. Clinical features of otolith organ-specific vestibular dysfunction. Clin Neurophysiol. 2018;129:238-45.

9. Kumar L, Thakar A, Thakur B, Sikka K. Sensitivity and specificity of clinical and laboratory otolith function tests. Otol Neurotol. 2017;38:e378-83.

10. Demirhan H, Yıldız M, Yiğit Ö. Do vestibular-evoked myogenic potential abnormalities in patients with Cochlear implant only reflect Saccular dysfunction? J Int Adv Otol. 2016;12:166-9.

11. Agrawal Y, Bremova T, Kremmyda O, Strupp M. Semicircular canal, saccular and utricular function in patients with bilateral vestibulopathy: analysis based on etiology. J Neurol. 2013;260:876-83.

12. Chisato F, Naoya E, Makoto K, Keiko S, Tatsuya Y, et al. Involvement of vestibular organs in idiopathic sudden hearing loss with vertigo: an analysis using OVEMP and CVEMP testing. Clin Neurophysiol. 2014;126:1033-8.

13. Bao XL, Xu H, Sun Q, Liu JH, Guo JL. Ocular vestibular evoked myogenic potentials of healthy young people. Zhongguo Er Bi Yan Hou Tou Jing Wai Ke Za Zhi. 2012;6:308-10.

14. Niu X, Zhang Y, Zhang Q, Xu X, Han P, Cheng Y, et al. The relationship between hearing loss and vestibular dysfunction in patients with sudden sensorineural hearing loss. Acta Otolaryngol. 2016;136:225-31.

15. Kitahara T, Takeda N, Nishiike S, Okumura S, Kubo T. Prognosis of inner ear periphery and central vestibular plasticity in sudden deafness with vertigo. Ann Otol Rhinol Laryngol. 2005;114:786-91.

16. Korres S, Stamatiou GA, Gkoritsa E, Riga M, Xenelis J. Prognosis of patients with idiopathic sudden hearing loss: role of vestibular assessment. J Laryngol Otol. 2011;125:251-7.

17. Ryu IS, Yoon TH, Ahn JH, Kang WS, Choi BS, Lee JH, et al. Threedimensional fluid-attenuated inversion recovery magnetic resonance imaging in sudden sensorineural hearing loss: correlations with audiologic and vestibular testing. Otol Neurotol. 2011;32:1205-9.

18. Shepard NT, Jacobson GP. The caloric irrigation test. Handb Clin Neurol. 2016;137:119-31.

19. Colebatch JG, Rosengren SM, Welgampola MS. Vestibular-evoked myogenic potentials. Handb Clin Neurol. 2016;137:133-55. 
20. Mudduwa R, Kara N, Whelan D, Banerjee A. Vestibular evoked myogenic potentials: review. J Laryngol Otol. 2010;124:1043-50.

21. Govender S, Rosengren SM, Dennis DL, Lim LJ, Colebatch JG. Contrasting phase effects on vestibular evoked myogenic potentials (VEMPs) produced by air- and bone-conducted stimuli. Exp Brain Res. 2016;234:141-9.

22. Papathanasiou ES, Murofushi T, Akin FW, Colebatch JG. International guidelines for the clinical application of cervical vestibular evoked myogenic potentials: an expert consensus report. Clin Neurophysiol. 2014;125:658-66.

Ready to submit your research? Choose BMC and benefit from:

- fast, convenient online submission

- thorough peer review by experienced researchers in your field

- rapid publication on acceptance

- support for research data, including large and complex data types

- gold Open Access which fosters wider collaboration and increased citations

- maximum visibility for your research: over $100 \mathrm{M}$ website views per year

At BMC, research is always in progress.

Learn more biomedcentral.com/submissions 